\title{
Antikoagulation auf schmalem Grat
}

\author{
Mit zunehmendem Alter steigt nicht nur die Prävalenz von Vorhofflimmern, sondern auch \\ die von Niereninsuffizienz. Patienten mit Niereninsuffizienz und Vorhofflimmern erleiden nicht \\ nur häufiger Schlaganfälle, sondern auch Blutungen. Deshalb stellt sich die Frage, \\ ob diese Patienten eine orale Antikoagulation bekommen sollten.
}

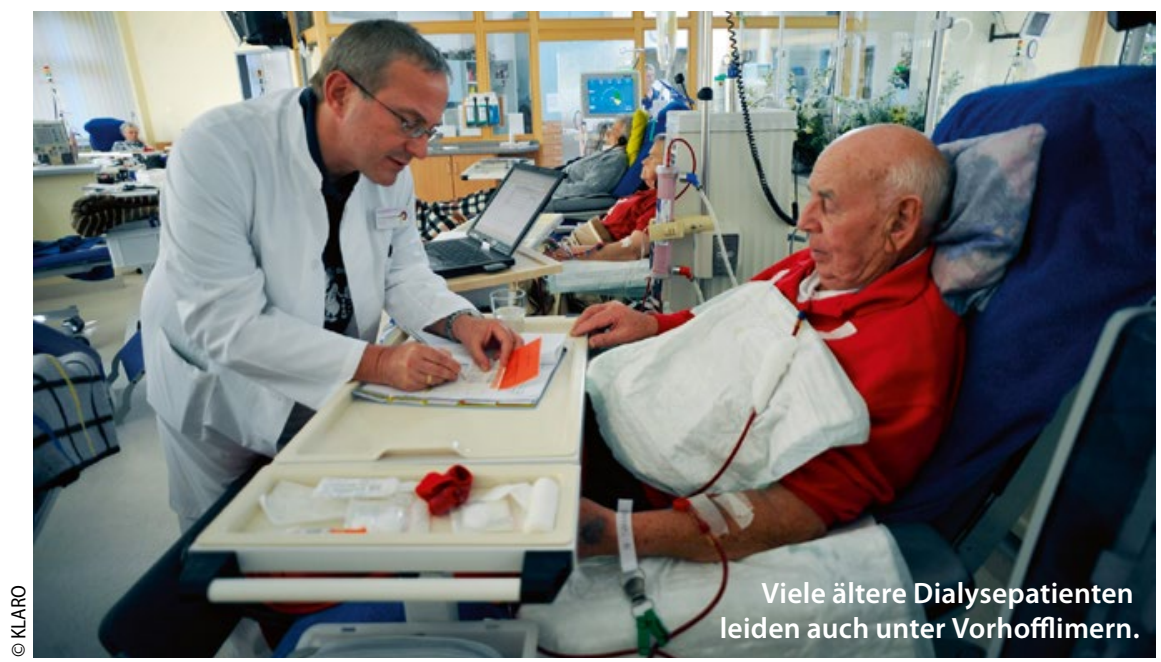

Fast jeder dritte Dialysepatient weist ein Vorhofflimmern auf. Eine große dänische Registerstudie ergab, dass Patienten mit Vorhofflimmern und Niereninsuffizienz ein doppelt so hohes Schlaganfallrisiko haben wie Patienten mit Vorhofflimmern ohne Niereninsuffizienz. Durch eine orale Antikoagulation kann dieses Risiko um zwei Drittel gesenkt werden. Allerdings ist auch das Blutungsrisiko dieser Patienten erhöht.

Der Netto-Effekt im dänischen Register: Patienten unter Antikoagulation erlitten seltener Ereignisse als Patienten ohne Antikoagulation. Die Sterblichkeit war in der Gruppe mit Antikoagulation signifikant um 15\% geringer. „Wer Antikoagulanzien bei diesen Patienten generell ablehnt, macht einen Fehler", folgerte Prof. Lars C. Rump, Direktor der neurologischen Universitätsklinik Düsseldorf. Man müsse allerdings die INR engmaschig kontrollieren, um das Blutungsrisiko zu minimieren. Rump empfahl wöchentliche Messungen und einen Zielbereich von 2,5-3.
ESC-Leitlinie hilft hier nicht weiter

Der $\mathrm{CHA}_{2} \mathrm{DS}_{2}$-VASc-Score ist umso höher, je nierenkränker die Patienten sind, erklärte Prof. Jan-Christoph Galle, Direktor der nephrologischen Klinik am Klinikum Lüdenscheid. Die zwei oder mehr Punkte im $\mathrm{CHA}_{2} \mathrm{DS}_{2}$-VASc-Score, die die ESC-Leitlinie für die Indikation zur Antikoagulation fordert, erfüllen niereninsuffiziente Patienten fast immer.

Sollen deshalb wirklich alle Patienten mit Vorhofflimmern Marcumar erhalten, obwohl sie auch Hochrisikopatienten für Blutungen sind? „Dieses Dilemma löst die ESC-Leitlinie leider nicht auf“, so Galle. Denn für Niereninsuffiziente gibt es keine einzige prospektive randomisierte kontrollierte Studie zur Schlaganfallprävention bei Vorhofflimmern.

In Deutschland erhält etwa die Hälfte der Dialysepatienten orale Antikoagulanzien. Das kann nur Marcumar sein, weil die neuen oralen Substanzen bei diesen Patienten kontraindiziert sind. Wie Galle betonte, gibt es auch Registerdaten, die zeigen, dass Vitamin-K-Antagonisten die
Schlaganfallrate sowie die Blutungsrate erhöhen und das Überleben verschlechtern.

Vitamin-K-Antagonisten und Gefäße Ein Grund für ungünstige Effekte von Vitamin-K-Antagonisten bei Nierenpatienten könnte die ausgeprägte Gefäßverkalkung sein, die durch diese Substanzen weiter verstärkt wird. Denn Vitamin K, das gebraucht wird, um die Gefäßverkalkung zu verhindern, wird antagonisiert. Man findet bei Nierenpatienten unter Marcumar einen höheren Verkalkungsgrad an Herzklappen und Koronarien. Aufgrund dieses Problems sollte man mit Vitamin-K-Antagonisten vorsichtig sein, wenn keine zwingende Indikation wie z. B. Klappenersatz vorliegt.

Dr. Angelika Bischoff

- DGIM-Kongress Mannheim 2015

\section{NOAK bei Vorhofflimmern und Niereninsuffizienz}

Patienten mit schwerer Niereninsuffizienz waren in den Zulassungsstudien für die neuen oralen Antikoagulanzien (NOAK) ausgeschlossen. Obwohl Apixaban, Rivaroxaban und Dabigatran sehr unterschiedlich renal eliminiert werden, sollte nach den offiziellen ESC-Empfehlungen ein NOAK bei schwerer Niereninsuffizienz mit einer $\mathrm{GFR}<30 \mathrm{ml} / \mathrm{min}$ nicht mehr eingesetzt werden. Bei einer moderaten Niereninsuffizienz mit einer GFR von 49-30 ml/min sollte Dabigatran nur in der niedrigen Dosierung von $2 \times 110$ $\mathrm{mg}$ täglich gegeben werden. Bei Rivaroxaban empfiehlt sich ab einer GFR $<50 \mathrm{ml} / \mathrm{min}$ auch eine reduzierte Dosis von $15 \mathrm{mg}$ einmal täglich. Bei Apixaban sollte die tägliche Dosis auf $2 \times 2,5$ mg reduziert werden, wenn 2 der folgenden 3 Kriterien vorliegen: Kreatinin > 1,5 mg/dl, Alter über 80 Jahre, Gewicht unter $60 \mathrm{~kg}$. Ob Dialysepflichtige Patienten von einer Antikoagulation profitieren, ist bisher nicht bewiesen. 